\title{
Multiple Positive Solutions for the Dirichlet Boundary Value Problems by Phase Plane Analysis
}

\author{
A. Kirichuka ${ }^{1}$ and F. Sadyrbaev ${ }^{2}$ \\ ${ }^{1}$ Daugavpils University, 13 Vienības Street, Daugavpils LV-5401, Latvia \\ ${ }^{2}$ Institute of Mathematics and Computer Science of University of Latvia, Raina Bulvaris 29, Riga LV-1469, Latvia \\ Correspondence should be addressed to A. Kirichuka; anita.kiricuka@du.lv
}

Received 1 July 2015; Accepted 28 September 2015

Academic Editor: Jaume Giné

Copyright (c) 2015 A. Kirichuka and F. Sadyrbaev. This is an open access article distributed under the Creative Commons Attribution License, which permits unrestricted use, distribution, and reproduction in any medium, provided the original work is properly cited.

\begin{abstract}
We consider boundary value problems for scalar differential equation $x^{\prime \prime}+\lambda f(x)=0, x(0)=0, x(1)=0$, where $f(x)$ is a seventh-degree polynomial and $\lambda$ is a parameter. We use the phase plane method combined with evaluations of time-map functions and make conclusions on the number of positive solutions. Bifurcation diagrams are constructed and examples are considered illustrating the bifurcation processes.
\end{abstract}

\section{Introduction}

Nonlinear boundary value problems for ordinary differential equations still form rapidly developed branch of classical analysis. The traditional issues like existence of solutions, uniqueness, and continuous dependence on boundary data are discussed in a number of classical and modern sources $[1,2]$. Less studied are complicated problems of the number of solutions as well as of their dependence on parameters. Of special value are results on existence of positive solutions due to multiple applications. We mention here the works [3-7], where two-point boundary value problems with parameters were considered for the second-order ordinary differential equations. The problem of finding multiple positive solutions was treated in [8]. Nonlinearities of polynomial type were considered in [9]. The time-map technique was applied for investigation of similar problems in [10].

Our goal in this note is to demonstrate how elementary phase plane analysis combined with evaluations of timemap functions can provide the researchers with significant information on the number and properties of solutions. We have chosen problem

$$
\begin{aligned}
x^{\prime \prime}+\lambda f(x) & =0, \\
x(0) & =0, \\
x(1) & =0,
\end{aligned}
$$

where $f(x)$ is a seventh-degree polynomial. Our technique is based on a phase plane analysis. To find positive solutions we will use the first zero function (the so called time-map function). By the first zero function we mean the mapping $\gamma \rightarrow t_{1}(\gamma)$, where $t_{1}(\gamma)$ is the first zero on the right of a solution of the Cauchy problem

$$
\begin{aligned}
x(0) & =0, \\
x^{\prime}(0) & =\gamma .
\end{aligned}
$$

The paper [11] discusses the cases of $f(x)$ being thirdand fifth-degree polynomials. It was observed that problem (1) may have, respectively, three or five positive solutions. We focus on the case of $f(x)$ being seventh-degree polynomial.

In Section 2, we provide basic facts about first zero functions. In Section 3, we formulate proposition about number of positive solutions for the Dirichlet boundary value problem. The example in Section 4 provides the detailed description of the respective time-map functions, solutions, and bifurcation diagrams. In the final section, we summarize the results and make conclusions.

\section{Basic Facts about Time-Map Function \\ Consider differential equation}

$$
x^{\prime \prime}+f(x)=0 .
$$


If $x(t, \gamma)$ is a solution of (3) with the initial conditions

$$
\begin{gathered}
x(0)=0, \\
x^{\prime}(0)=\gamma,
\end{gathered}
$$

then we denote by $t_{1}(\gamma)$ the first zero function (time-map) for Cauchy problem (3), (4).

Consider the problem with a parameter

$$
\begin{aligned}
X^{\prime \prime}+\lambda f(X) & =0, \\
X(0) & =0, \\
X^{\prime}(0) & =\alpha
\end{aligned}
$$

and denote the first zero function $U(\alpha, \lambda)$.

The relation between these two time-map functions was established previously [11, 12]:

$$
U(\alpha, \lambda)=\frac{1}{\sqrt{\lambda}} t_{1}\left(\frac{\alpha}{\sqrt{\lambda}}\right) .
$$

If $x(t ; \gamma)$ is a solution of the Cauchy problem (3), (4), then

$$
X(t ; \alpha)=x\left(\sqrt{\lambda} t ; \frac{\alpha}{\sqrt{\lambda}}\right)
$$

solves the initial value problem (5). For details consult [12].

\section{Nonlinearity with Seventh-Degree Polynomial}

In the sequel, we consider the problem with $f(x)$ the seventhdegree polynomial as follows:

$$
x^{\prime \prime}=\lambda f(x), \quad \lambda>0
$$

where

$$
\begin{gathered}
f(x)=(x-a)(x-b)(x-c)(x-d)(x-e)(x-g) \\
\cdot(x-h), \quad 0<a<b<c<d<e<g<h
\end{gathered}
$$

with the Dirichlet conditions

$$
\begin{aligned}
& x(0)=0, \\
& x(1)=0 .
\end{aligned}
$$

Let function $F(x)$ be the primitive function of $f(x)$

$$
F(x)=\int_{0}^{x} f(s) d s
$$

and let the conditions

$$
F(a)<F(c)<F(e)<F(h)
$$

be fulfilled.

Let us consider phase plane for (8). The value of the first zero function at $\alpha$ is the time needed to move from a point $(0, \alpha)$ to the first intersection point with the $X^{\prime}$-axis. Denote

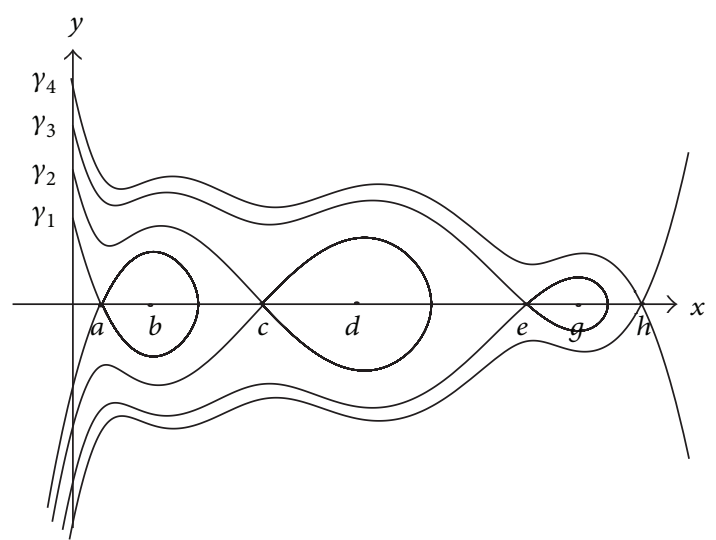

FIgURE 1: The phase portrait of (14).

by $X(t, \alpha)$ a solution of the Cauchy problem (5). A set of $(\lambda, \alpha)$ such that $(\lambda, X(t, \alpha))$ solves the Dirichlet problem $(8)$, (10) will be called a solution curve. All such $(\lambda, \alpha)$ satisfy the equality

$$
U(\alpha, \lambda)=1
$$

The phase portrait for (8) has 7 critical points; 3 of them are the points of type "center" and 4 are points of type "saddle": $(a, 0),(c, 0),(e, 0)$, and $(h, 0)$.

Consider equation

$$
\begin{aligned}
x^{\prime \prime} & =f(x)=(x-a)(x-b)(x-c)(x-d)(x-e) \\
& \cdot(x-g)(x-h) .
\end{aligned}
$$

Suppose that $\gamma_{1}, \gamma_{2}, \gamma_{3}$, and $\gamma_{4}$ are initial values such that trajectories $\left(X\left(t, \gamma_{i}\right), X^{\prime}\left(t, \gamma_{i}\right)\right)$ enter the saddle points (as in Figure 1).

Part of the phase portrait for (14) has the form shown in Figure 1.

We will look now for solutions of the Dirichlet problem (14), (10) choosing the initial conditions in one of the four intervals separately $\gamma \in\left(0, \gamma_{1}\right),\left(\gamma_{1}, \gamma_{2}\right),\left(\gamma_{2}, \gamma_{3}\right),\left(\gamma_{3}, \gamma_{4}\right)$.

We note that phase portraits of (8) and (14) are equivalent (critical points are the same and trajectories are in one-toone correspondence due to formula (7)). If we change $\lambda$ then the initial values for trajectories entering saddle points change also as $\alpha_{i}=\sqrt{\lambda} \gamma_{i}(i=1,2,3,4)$.

Proposition 1. For any $\lambda>0$, there exists $\gamma \in\left(0, \gamma_{1}(\lambda)\right)$ such that $T(\gamma)=1$, where $T(\gamma)$ is the time-map function for (14).

Proof. Let $x\left(t, \gamma_{1}\right)$ be a solution which goes to the saddle point at $x=a$. Consider solutions $x(t, \gamma)$ for $0<\gamma<\gamma_{1}$ where $T(\gamma)$ is the time needed for a point to move along phase trajectory from point $(0, \gamma)$ to point $(0,-\gamma) . T(\gamma)$ tends to zero as $\gamma$ goes to zero (since $(0,0)$ is not a critical point) and $T(\gamma)$ tends to $+\infty$ as $\gamma$ goes to $\gamma_{1}$. By continuity of $T(\gamma)$ there exists $\gamma \in$ $\left(0, \gamma_{1}\right)$ such that $T(\gamma)=1$.

Proposition 2. For the first zero function $T(\gamma)$ of (14) in each of the intervals $\left(\gamma_{1}, \gamma_{2}\right),\left(\gamma_{2}, \gamma_{3}\right)$, and $\left(\gamma_{3}, \gamma_{4}\right)$ there exist $T_{i \min }=$ $\min _{\gamma_{i}<\gamma<\gamma_{i+1}} T(\gamma), i=1,2,3$, respectively. 


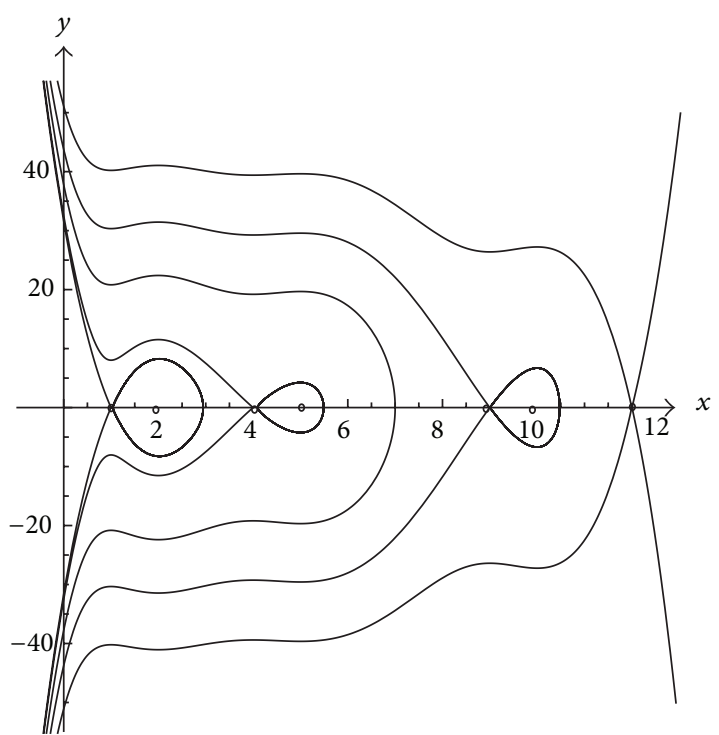

FIgURE 2: The phase portrait of (15), $\lambda=0.0343$.

Proof. Consider solutions $x(t, \gamma)$ for $\gamma_{1}<\gamma<\gamma_{2}$ where $T(\gamma)$ is the time needed for a point to move along phase trajectory from point $(0, \gamma)$ to point $(0,-\gamma)$. Then $T(\gamma)$ tends to $+\infty$ as $\gamma$ goes to $\gamma_{1}$ and $T(\gamma)$ tends to $+\infty$ as $\gamma$ goes to $\gamma_{2}$. Function $T(\gamma)$ is continuous since the right side of (14) is a polynomial and there is continuous dependence of solutions on initial data. Therefore, there is a minimum of $T_{1 \text { min }}$ in $\left(\gamma_{1}, \gamma_{2}\right)$ at some $\gamma_{1 \text { min }}$ and, in this case also, the time function has a " $U$ " shaped graph, similarly to $T_{2 \min }$ and $T_{3 \min }$.

Remark 3. The graph of the time-map function has U-shaped segments and the number of positive solutions may be one, two, or more solutions depending on whether the graph of $T(\gamma)$ has one, two, or more intersections with the unity level (the unity refers to the length of the interval).

Proposition 4. There exists $\Lambda>0$ such that $\lambda>\Lambda$ the Dirichlet problem (8), (10) has at least seven solutions.

Proof. It follows from Propositions 1 and 2 and formula (6), where $t_{1}(\alpha)$ is the time-map function for equation $x^{\prime \prime}=f(x)$ and $f(x)$ is given in (9).

Remark 5. The relative positions of $T_{i \min }, i=1,2,3$, do not change if $\lambda$ tends to $+\infty$. This follows from (6).

Remark 6. Precise number of solutions of problem (8), (10) depends on convexity of time-map function $U(\alpha, \lambda)$ with respect to $\alpha$ and this can be checked using criteria for $\partial U / \partial \alpha$ and $\partial^{2} U / \partial \alpha^{2}$ as in [13].

\section{Examples}

\subsection{Example 1. Consider equation}

$$
\begin{aligned}
x^{\prime \prime}= & \lambda(x-1)(x-2)(x-4)(x-5)(x-9)(x-10) \\
& \cdot(x-12) .
\end{aligned}
$$

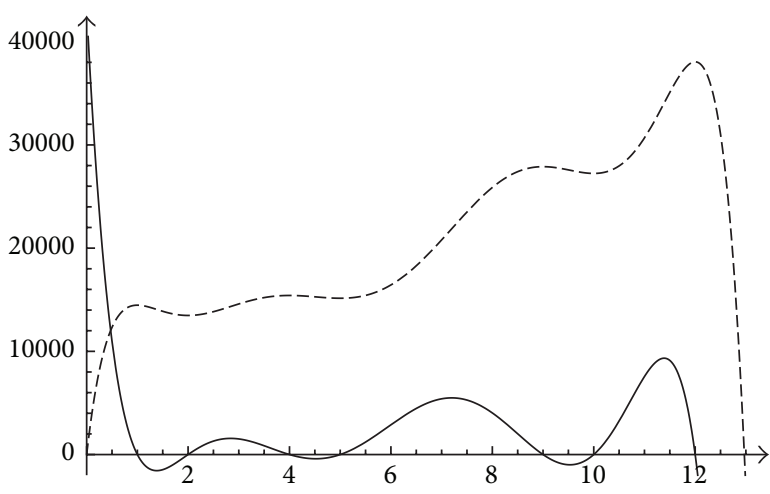

Figure 3: Graphs of the function $f(x)$ and $F(x)$ (dashed).

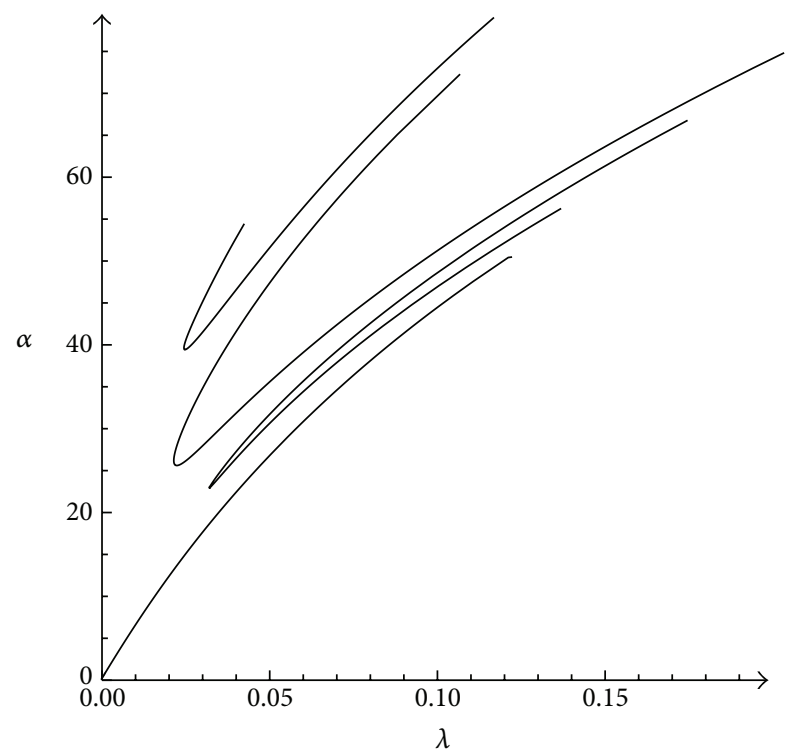

FIgURE 4: The bifurcation diagram for Example 1.

The phase portrait of (15) has 7 critical points; 3 of them are points of type "center" and 4 are points of type "saddle": $(1,0)$, $(4,0),(9,0)$, and $(12,0)$ (see Figure 2).

Then

$$
\begin{aligned}
f(x)= & (x-1)(x-2)(x-4)(x-5)(x-9)(x-10) \\
& \cdot(x-12) .
\end{aligned}
$$

The condition

$$
F(1)<F(4)<F(9)<F(12)
$$

is fulfilled (see Figure 3). Next we bring all the time-maps together and as a result get the bifurcation diagram in Figure 4.

The graphs of $U(\alpha, \lambda)$ for seven values of $\lambda$ are depicted in Figures 5-18.

\subsection{Example 2. Consider equation}

$$
x^{\prime \prime}=\lambda(x-1)(x-2)(x-5)(x-6)(x-8)(x-10)
$$

$$
\cdot(x-12) \text {. }
$$




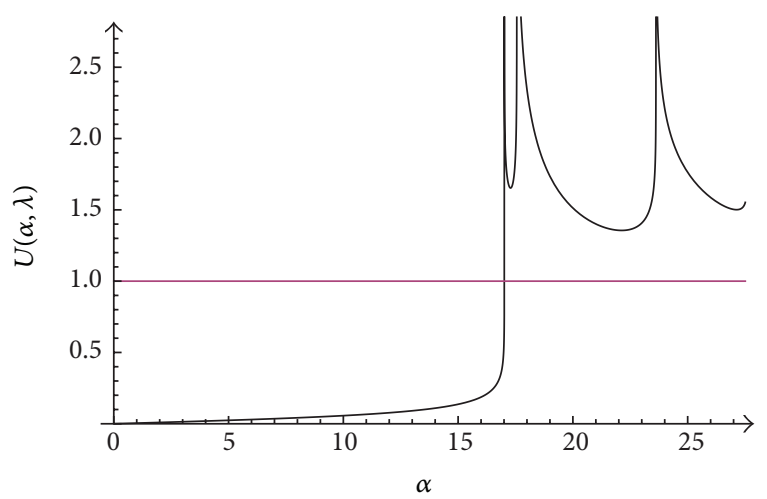

FIgURE 5: The graph of the function $U(\alpha, 0.01)$.

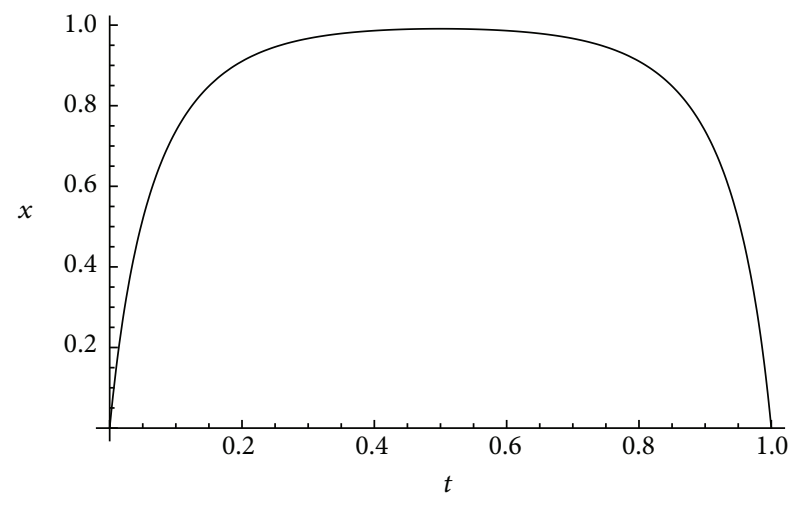

Figure 6: The solution of problem (15), (10), $\alpha \approx 17.0132$.

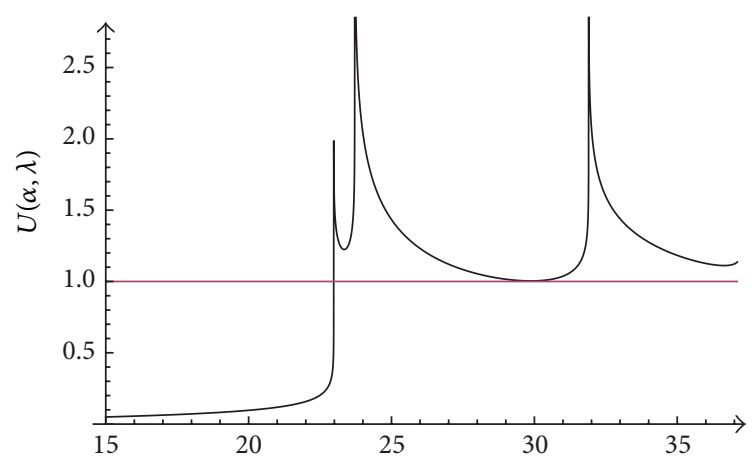

Figure 7: The graph of the function $U(\alpha, 0.01824)$.

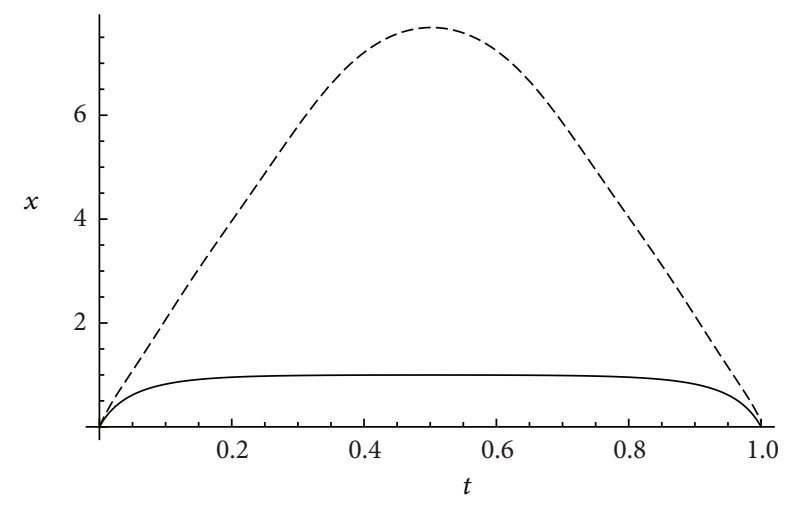

Figure 8: Two solutions of problem (15), (10), $\alpha \approx 22.9775, \alpha \approx$ 29.8788 .

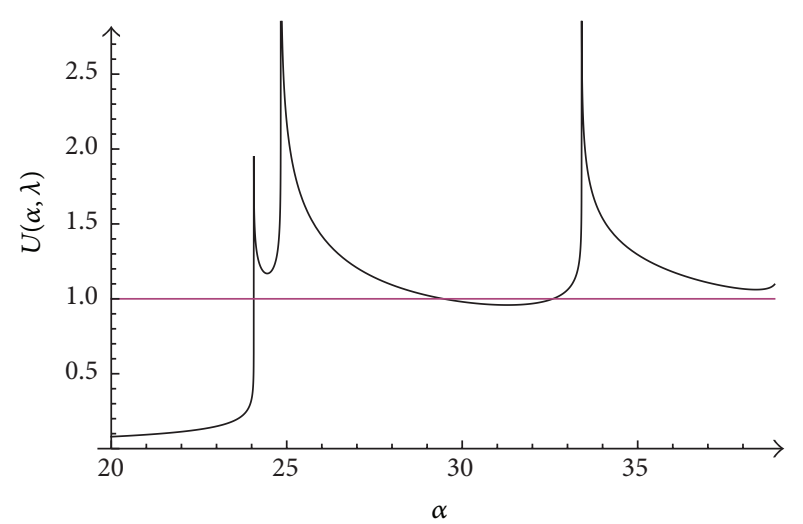

FIGURE 9: The graph of the function $U(\alpha, 0.02)$.

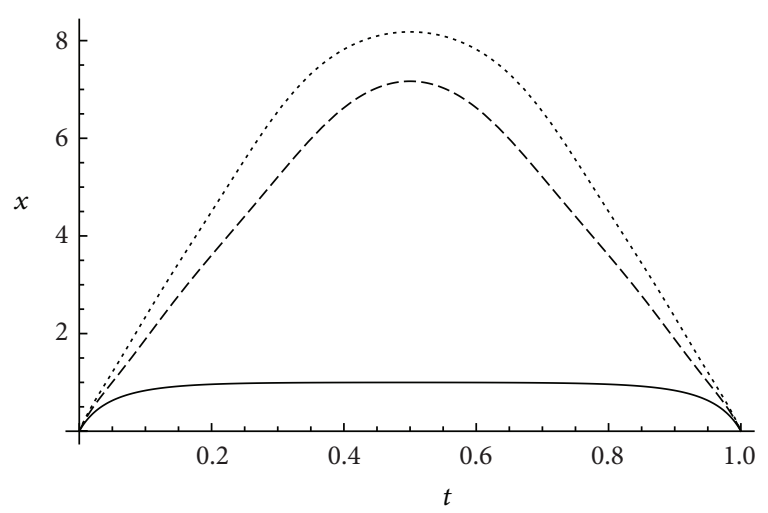

FIGURE 10: Three solutions of problem (15), (10), $\alpha \approx 24.0606, \alpha \approx$ 29.4663 , and $\alpha \approx 32.5832$.

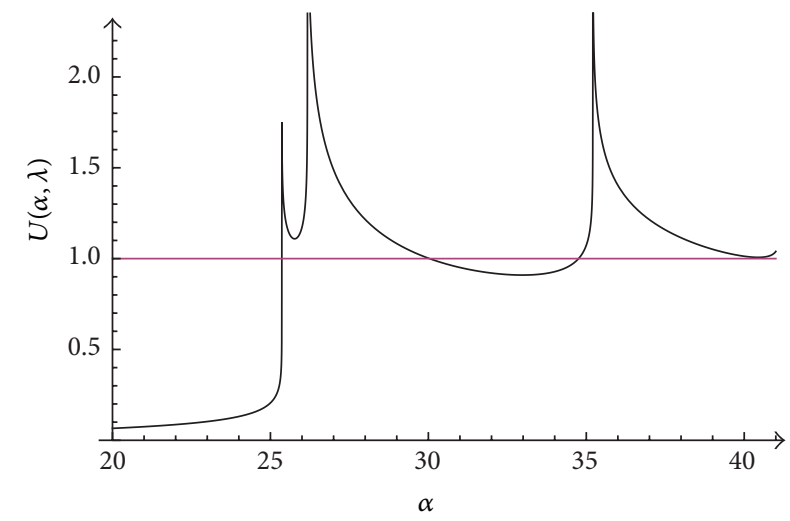

FIGURE 11: The graph of the function $U(\alpha, 0.02222)$.

The 012B phase portrait of (18) has 7 critical points; 3 of them are the points of type "center" and 4 are points of type "saddle": $(1,0),(5,0),(8,0)$, and $(12,0)$. The structure of the phase portrait for (18) is the same as that for (15).

Then

$$
\begin{aligned}
f(x)= & (x-1)(x-2)(x-5)(x-6)(x-8)(x-10) \\
& \cdot(x-12) .
\end{aligned}
$$




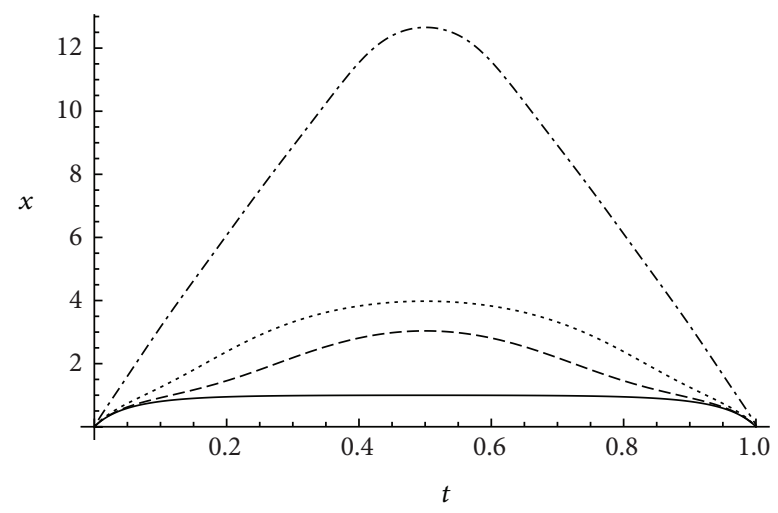

FIGURE 12: Four solutions of problem (15), (10), $\alpha \approx 25.3608, \alpha \approx$ $30.0365, \alpha \approx 34.7552$, and $\alpha \approx 41.4674$.

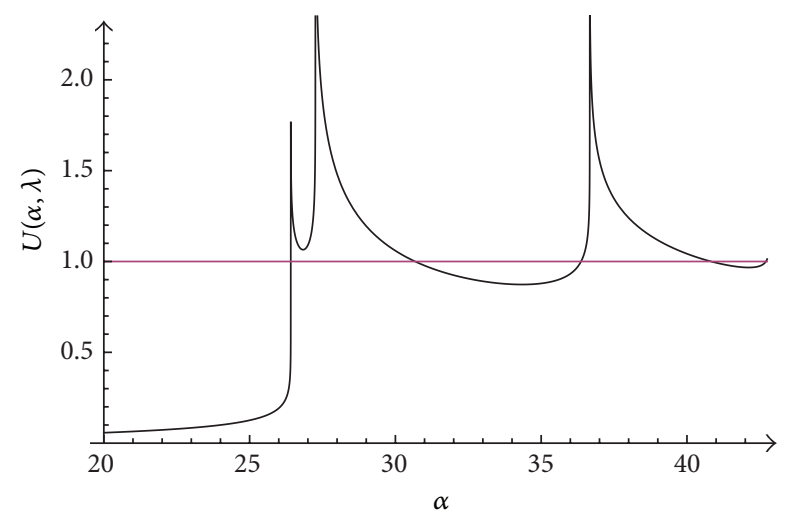

FIGURE 13: The graph of the function $U(\alpha, 0.0241)$.

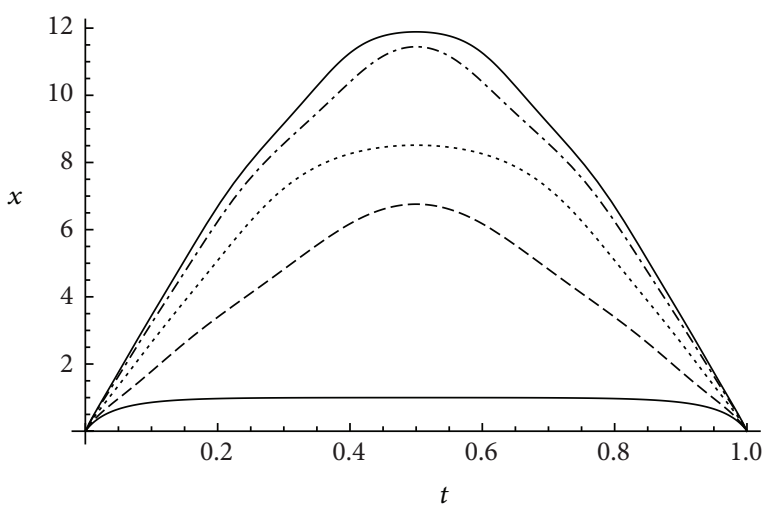

Figure 14: Five solutions of problem (15), (10), $\alpha \approx 26.4119, \alpha \approx$ $30.6763, \alpha \approx 36.3664, \alpha \approx 40.8286$, and $\alpha \approx 42.7074$.

The condition for the saddle points

$$
F(1)<F(5)<F(8)<F(12)
$$

is fulfilled also.

If we look at the bifurcation diagram in Figure 19, we see that there is no such value $\lambda$ where at some $\alpha$ the Dirichlet problem (18), (10) has seven solution because the difference between minimum values of the time-map function is large enough.

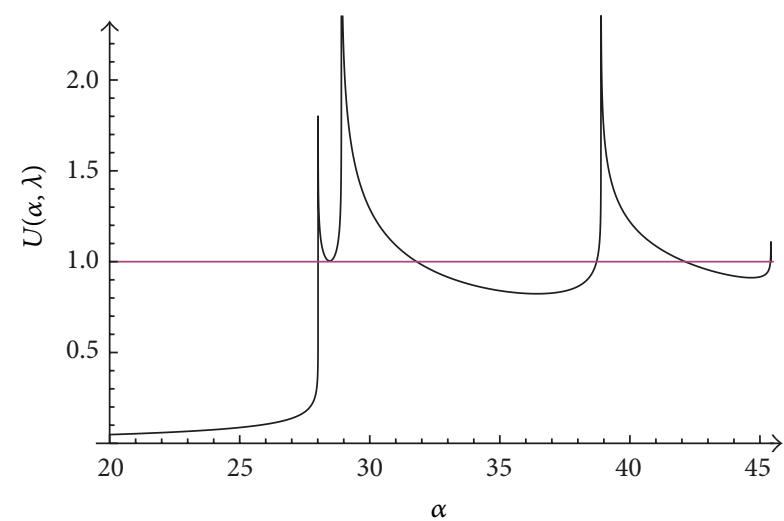

FIGURE 15: The graph of the function $U(\alpha, 0.02711)$.

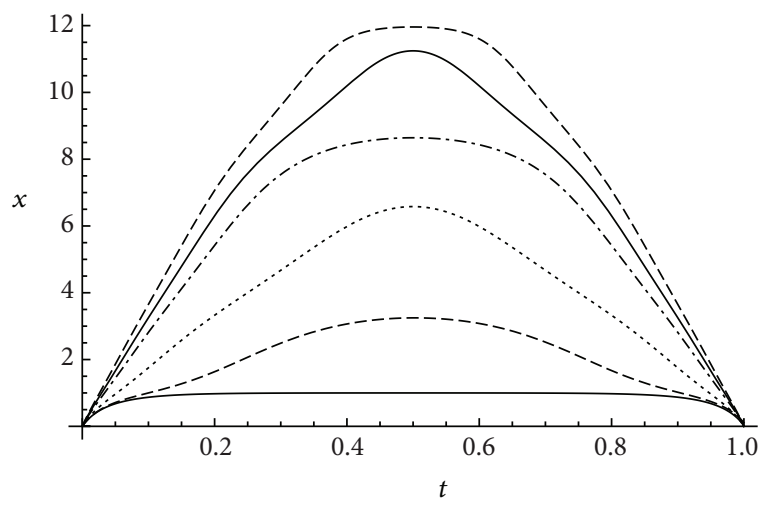

FIgURE 16: Six solutions of problem (15), (10), $\alpha \approx 28.0128, \alpha \approx$ 28.4601, $\alpha \approx 31.8026, \alpha \approx 38.7197, \alpha \approx 42.1239$, and $\alpha \approx 45.4026$.

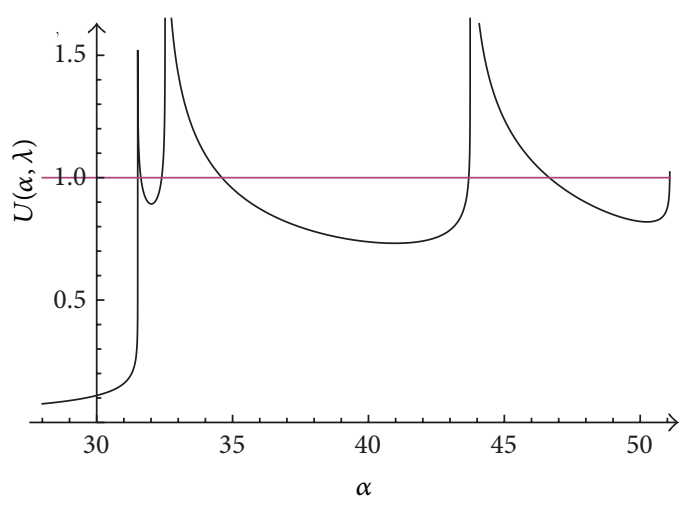

FIGURE 17: The graph of the function $U(\alpha, 0.0343)$.

\section{Conclusions}

For a polynomial of the type (9) but with arbitrary large odd number of zeros $k$ in presence of the condition $F\left(a_{1}\right)<$ $F\left(a_{3}\right)<\cdots<F\left(a_{k}\right)\left(a_{i}\right.$ are the zeros) there always exists large enough $\lambda$ such that the respective boundary value problem (1) has at least $k$ positive solutions.

If $\lambda$ is not large then in presence of the condition $F\left(a_{1}\right)<$ $F\left(a_{3}\right)<\cdots<F\left(a_{k}\right)$ the number of solutions of the boundary value problem (1) depends on the relative positions of minima 


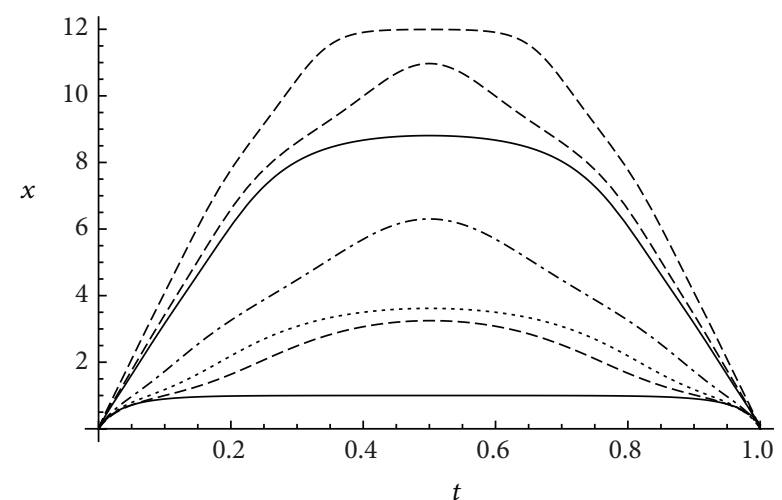

FIgURE 18: Seven solutions of problem (15), (10), $\alpha \approx 31.5093, \alpha \approx$ $31.6248, \alpha \approx 32.3915, \alpha \approx 34.6074, \alpha \approx 43.6897, \alpha \approx 45.7099$, and $\alpha \approx 51.0893$.

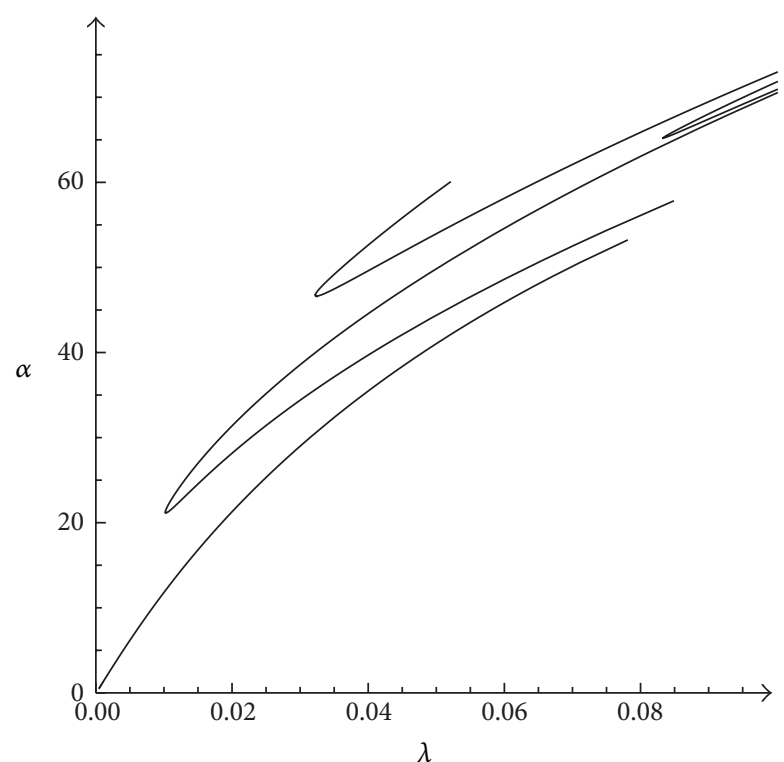

FIGURE 19: The bifurcation diagram for Example 2.

of the first zero function (time-map) $T(\gamma)$. These relative positions may be regulated by the choice of parameters $a_{1}, a_{2}, \ldots, a_{k}$

\section{Conflict of Interests}

The authors declare that there is no conflict of interests regarding the publication of this paper.

\section{References}

[1] S. R. Bernfeld and V. Lakshmikantham, An Introduction to Nonlinear Boundary Value Problems, Academic Press, New York, NY, USA, 1974.

[2] Y. A. Klokov and N. I. Vasilyev, Foundations of the Theory of Nonlinear Boundary Value Problems, Zinatme, Riga, Latvia, 1978 (Russian).

[3] T. Shibata, "Asymptotic shape of solutions to nonlinear eigenvalue problems," Electronic Journal of Differential Equations, vol. 2005, no. 37, pp. 1-16, 2005.
[4] T. Shibata, "Inverse spectral problems for nonlinear SturmLiouville problems," Electronic Journal of Differential Equations, vol. 2007, no. 74, pp. 1-10, 2007.

[5] T. Shibata, "Multiparameter variational eigenvalue problems with indefinite nonlinearity," Canadian Journal of Mathematics, vol. 49, no. 5, pp. 1066-1088, 1997.

[6] S. Tanaka, "On the uniqueness of positive solutions for twopoint boundary value problems of Emden-Fowler differential equations," Mathematica Bohemica, vol. 135, no. 2, pp. 189-198, 2010.

[7] S. Tanaka, "On the uniqueness of solutions with prescribed numbers of zeros for a two-point boundary value problem," Differential and Integral Equations, vol. 20, no. 1, pp. 93-104, 2007.

[8] M. Gaudenzi, P. Habets, and F. Zanolin, "A seven-positive-solutions theorem for a superlinear problem," Advanced Nonlinear Studies, vol. 4, no. 2, pp. 149-164, 2004.

[9] J. Smoller and A. Wasserman, "Global bifurcation of steadystate solutions," Journal of Differential Equations, vol. 39, no. 2, pp. 269-290, 1981.

[10] W. Dambrosio, "Time-map techniques for some boundary value problems," Rocky Mountain Journal of Mathematics, vol. 28, no. 3, pp. 885-926, 1998.

[11] S. Atslega and F. Sadyrbaev, "Multiplicity of solutions for the Dirichlet problem: comparison of cubic and quintic cases," Proceedings of IMCS of University of Latvia, vol. 11, pp. 73-82, 2011, http://www.lumii.lv/resource/show/214.

[12] A. Gritsans and F. Sadyrbaev, "Nonlinear spectra for parameter dependent ordinary differential equations," Nonlinear Analysis: Modelling and Control, vol. 12, no. 2, pp. 253-267, 2007.

[13] A. Gritsans and F. Sadyrbaev, "Time map formulae and their applications," Proceedings of IMCS of University of Latvia, vol. 8, pp. 23-34, 2008, http://www.lumii.lv/Pages/LUMII-2008/ contents.htm. 


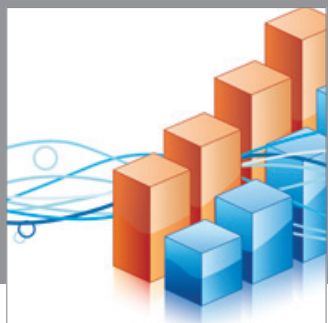

Advances in

Operations Research

mansans

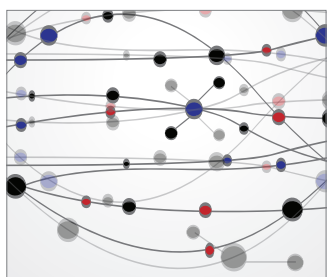

The Scientific World Journal
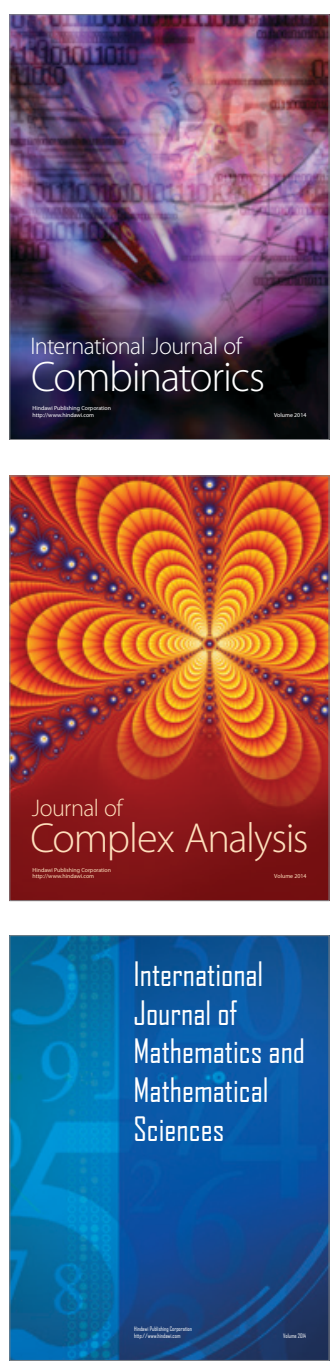
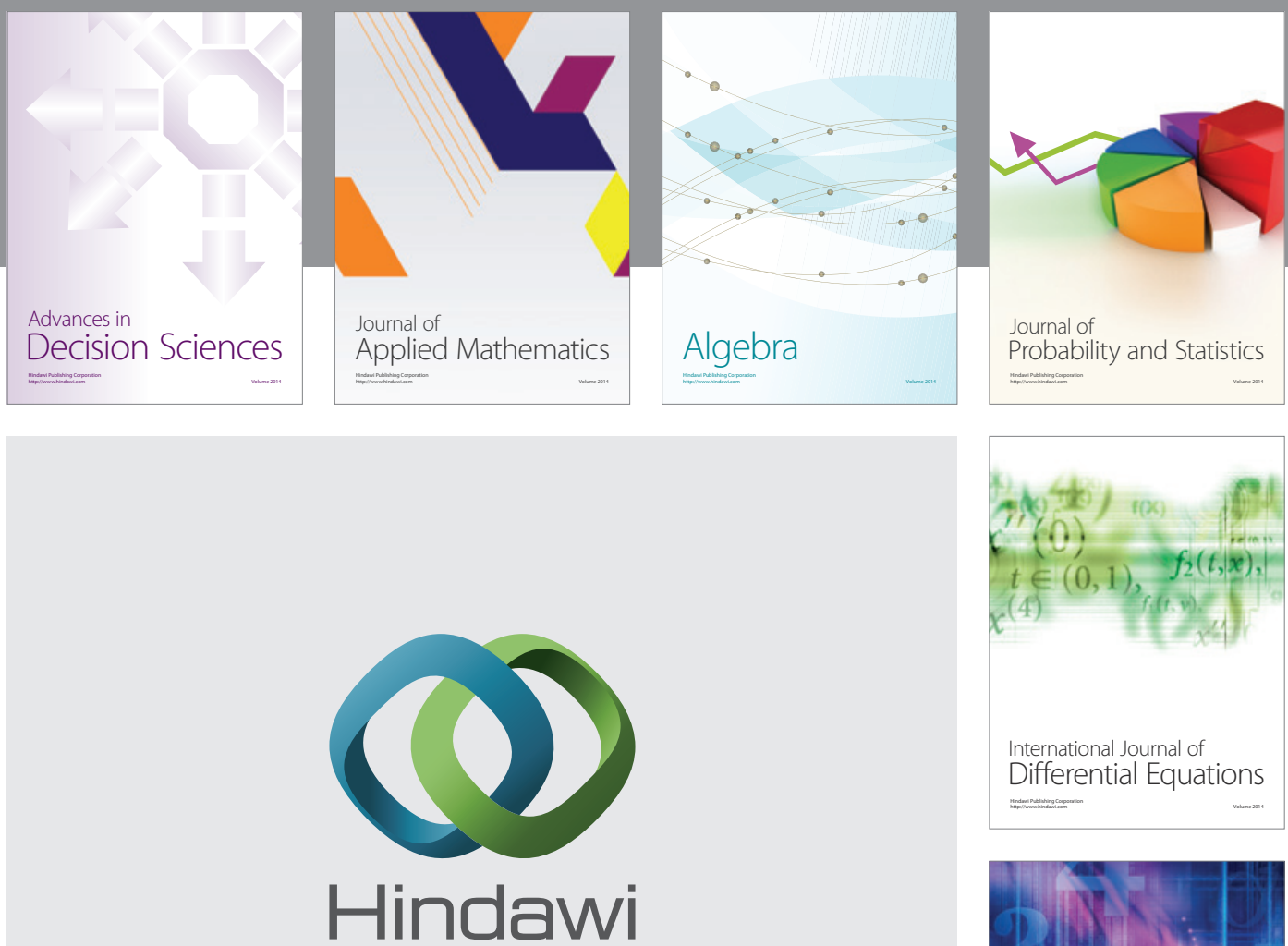

Submit your manuscripts at http://www.hindawi.com
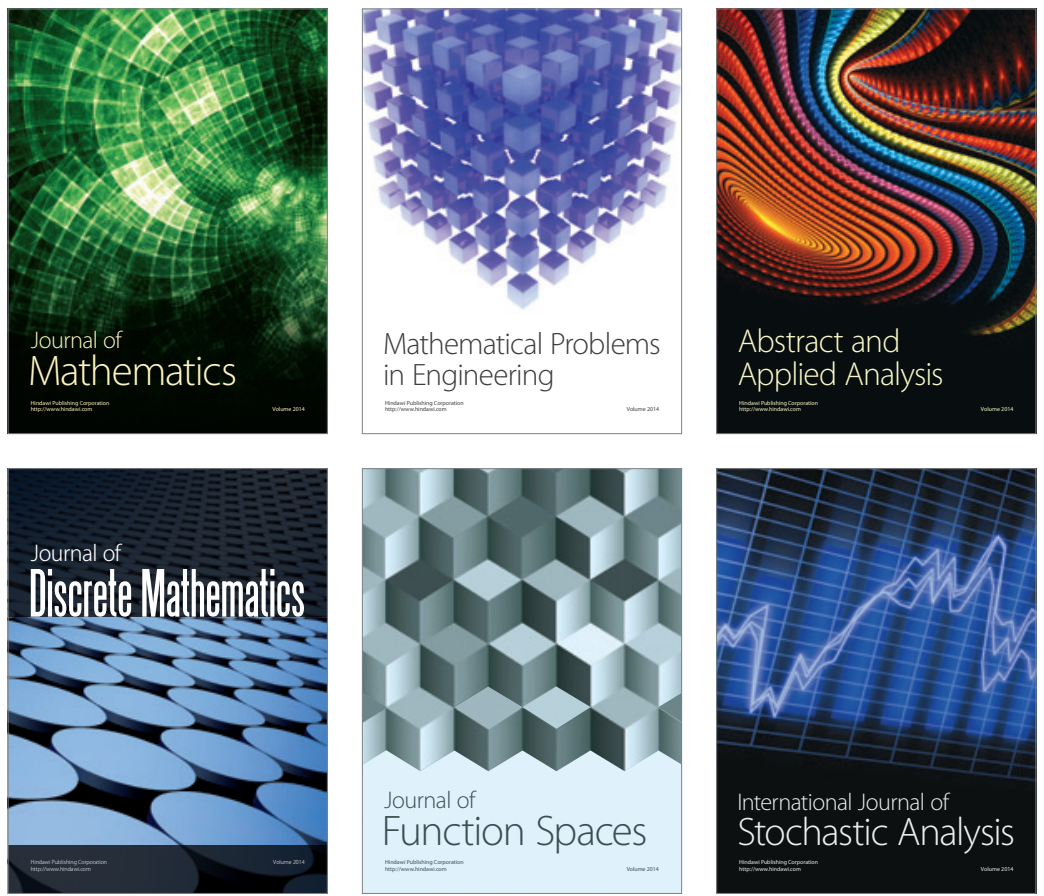

Journal of

Function Spaces

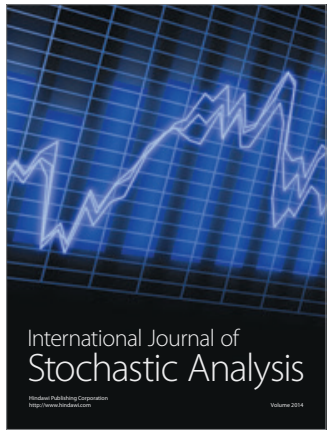

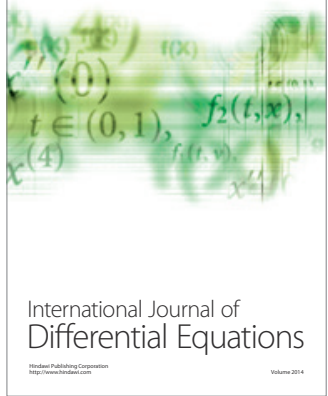
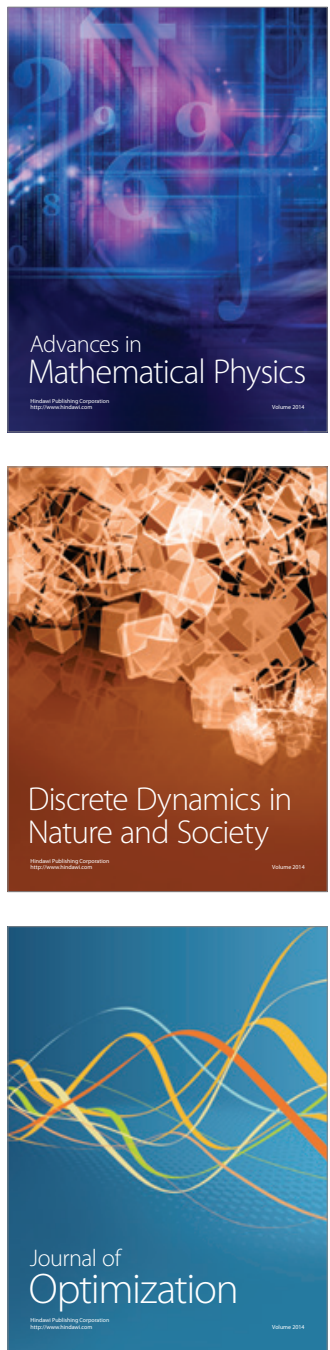